\title{
Die Kaumuskulatur der Anuren (Bufo vulgaris japonicus) während der Metamorphose.
}

\author{
Von \\ Akoyosi Takisawa, Yosio Ōhara und Koichiro Kanō. \\ (Aus dem Anatomischen Institut der Gun-ma Universität zu Maebashi.) \\ Mit 11 Textfiguren.
}

\section{Einleitung.}

Als Resultate einer durch Lupenpräparation und Schnittuntersuchung einander ergänzten Mosaikarbeit sind wir zum Schlusse gekommen, daß die Kaumuskeln der Kröte während der Metamorphose sich in zweierlei Weisen verändern: die larvalen Kaumuskeln werden währenddessen durch die neuen ersetzt, deren Muskelfasern von den $z$ wischen denjenigen der larvalen Muskeln befindlichen Fibroblasten abstammen, während die larvalen Muskelfasern selbst zugrunde gehen; anderseits entwickelt sich während der Metamorphose ein Kaumuskel aus den Mesenchymzellen, die mit den larvalen Muskelfasern in keinem Zusammenhang stehen.

Die Kaumuskeln der Anuren im erwachsenen und larvalen Zustand sowie während der Metamorphose sind bekanntlich seit der Mitte des vorigen Jahrhunderts von vielen Autoren studiert worden. Unter ihnen hat A. Luther (1914) dieselbe in seinem meisterhaften Werk „über die vom N. trigeminus versorgte Muskulatur der Amphibien " besonders eingehend beschrieben. Er hat sie aber nur auf präparatorischem Weg untersucht.

Die Rück- bezw. Neubildung der Muskelfasern während der Entwicklung sind auch von vielen Forschern bei verschiedenen Tierarten bestätigt. Aber bezüglich der Neubildung der Muskelfasern unter gleichzeitiger Rückbildung der alten liegt, soweit uns bekannt, nur die einzige Mitteilung von Helene Müller (1921) vor. Nach ihr bestehen die morphologischen Sonderungsvorgänge, welche bei Anuren während der Metamorphose sich in der dorsalen Rumpfmuskulatur abspielen, 
hauptsächlich in der Rück- und Neubildung von Muskelfasern. Sie glaubte dabei, da $B$ die neuen Muskelfasern aus den alten durch Spaltung entstehen, was sie aber nicht beweisen konnte.

Takisawa und Sunaga (1951) haben die Entwicklung des M. depressor mandibulae während der Metamorphose in der Hauptsache bei der Kröte studiert; $z$ wischen den Muskelfasern des Orbitohyoideus, eines der 5 Facialismuskeln der Anurenlarven, die unter dem Namen Levator hyoidei zusammengefaßt sind, erscheinen die neuen Myoblasten, welche im Laufe der Metamorphose den Depressor mandibulae des erwachsenen Tieres bilden, während der Orbitohyoideus selbst und die übrigen 4 Muskeln auch zugrunde gehen. Mit Thioninfärbung konnten sie ferner feststellen, daß diese neuen Myoblasten von den Fibroblasten des Endomysiums des Orbitohyoideus stammen.

\section{Material und Methode.}

Für unsere Versuche haben wir ausschließlich die Kröte (Bufo vulgaris japonicus) benutzt, da ihre Larven bekanntlich viele Vorteile darbieten. Vor allem sind sie wesentlich kleiner, was freilich für Mikrodissektion etwas nachteilig, so doch für zytologischen $Z$ weck sehr bequem ist. Ferner sind die aus einem Laich gezüchteten Krötenlarven in jeder Entwicklungsstufe voneinander nicht so sehr abweichen, wie andere Anurenlarven. Die im etwa $18^{\circ} \mathrm{C}$ Wasser gezüchteten Larven wurden bis zum Abschluß der Metamorphose von Tag zu Tag fixiert. Vom Verschluß des Operculums bis zum Durchtritt der vorderen Extremitäten, dauert es etwa 5 Wochen. Die Züchtung war weiter bis zum Ende der Metamorphose etwa eine Woche möglich.

Die erwachsenen Kröten und die in Formol fixierten oder mit Hämatoxylin (Hansen) in toto gefärbten Larven wurden unter Binokularlupe (10-100x) präpariert. Wir konnten dadurch die Orientierung für die nachfolgenden Schnittuntersuchungen gewinnen.

Die Materialien wurden je nach dem $Z$ weck in verschiedene Fixierungsflüssigkeiten eingetaucht: Levi, Bouin, Regaud, Zenker.Formol und Formol-Alkohol. Einbettung in Paraffin; Zerlegung in 4-5 $\mu$ Serienschnitte; Färbung je nach dem $Z$ weck. Es wurde dabei festgestellt, daß für verschiedene Zelleinschlüsse die Fixierung nach Levi und die Färbung mit Heidenhain's Eisenhämatoxylin am besten geeignet sind. Für die Herstellung der Übersichtspräparate wurde die Fixierung mit Zenker-Formol mit Nachchromierung, mit Regaud mit Nachchromierung oder mit Bouin, und die Färbung mit Hämatoxylin 
Die Kaumuskulatur der Anuren (Bufo vulgaris japonicus) während der Metamorphose. 3

(Hansen)-Eosin, mit Hejdenhain's Eisenhämatoxylin oder mit Azan angewandt. Besonders empfehlenswert fanden wir die Fixierung mit Zenker-Formol mit Nachchromierung.

Für die Untersuchung der basophilen Substanz wurden die mit Zenker-Formol oder Formol-Alkohol fixierten Schnitte einfach mit Thionin gefärbt und in Wintergrünöl beobachtet.

Für die Untersuchung des Glykogens wurden die mit ZenkerFormol fixierten Schnitte durch Bauersche Methode nach Vorbehandlung mit $2 \%$ Chromsäure oder mit $0.5 \%$ Überjodsäure (periodic acid Schiff's reaction) tingiert; die Speichelreaktion wurde auch geprüft.

Für die Untersuchung der Kerne wurden die in Formol-Alkohol oder in Zenker-Formol fixierten Schnitte durch Feulgensche Nuklealreaktion gefärbt.

\section{Die Kaumuskulatur der erwachsenen Kröte und der Krötenlarve.}

Bevor wir die Umgestaltungen der Kaumuskulatur während der Metamorphose studieren, war uns zweckmäßig, sie an erwachsenen Kröten und Krötenlarven $\mathrm{zu}$ untersuchen.

A. Die Kaumuskulatur der erwachsenen Kröte (Figg. 1, 2).

Am Adductor mandibulae der erwachsenen Anuren unterscheidet Luther (1914) folgende 6 Muskeln:

a) lateral vom Hauptstamm des N. V-3 und vom N. V-2 :

1) Adductor mandibulae externus ( $M$. masseter major Gaupps).

b) caudal (bezw. mediocaudal) vom Hauptstamm des N. V-3 (Add. mand. posterior):

2) Add. mand. post. subexternus.

3) Add. mand. post. lateralis (M. masseter minor Gaupps).***

4) Add. mand. post. longus (M. temporalis der Autoren).

5) Add. mand. post. articularis (kurzer Kopf des Temporalis Gaupp's).***

* Wir folgen hier die Benennung der Kaumuskeln nach Luther (1914) und L u bosch (1938), nicht nach Edgeworth (1935), da die Benennung des letztgenannten Autors zwar theoretisch besser, doch noch nicht eingebürgert ist.

** Die Synonyme der Kaumuskeln sind in L uther, L u bosch (1915) und Edgeworth ausführlich angegeben.

*** Lubosch (1915) verteilt und benennt die Kaumuskeln der erwachsenen Anuren anders als $L$ uther. Wir können aber nicht verstehen, warum er dabei Masseter minor Gaupps mit Add. mand. post. articularis Luthers identifiziert, ferner warum er Add. mand. post lat. Luthers weglässt. Er schlieBt sich aber später (1938) an Luther an, 
c) medial von den $\mathrm{Nn} . \mathrm{V}-2$ und $\mathrm{V}-3$, zwischen diesen und dem $\mathrm{N}$. V-1 (Add. mand. internus) :

6) M. pterygoideus (M. pterygoideus Gaupps).

Man findet bei Luther eingehende Beschreibung für all diese Muskeln. Insofern es aber die Kröte betrifft, sei noch folgendes hinzufügen.

1) Add. mand. externus ( $a$ in Figuren). Wie Luther beschreibt, entspringt er vom ventralen Rand des Processus zygomaticus (Prz) des Squamosum ( $S q)$ (Tympanicum Gaupps) sowie von der Innenfläche des rostro ventralen Quadranten des knorpeligen Annulus tympanicus $(A n n)$, und zieht medial vom Quadratomaxillare zum Unterkiefer $(M)$, wo er dorsal und lateral am knorpeligen Mandibulare sowie an der lateralen Seite des Goniale inseriert.

2) Add. mand. post. subexternus (aps). Nach Luther soll er bei Bufo fehlen. Wir finden ihn dagegen bei Bufo als einen sehr schmalen Strang, den man sehr leicht übersieht. Sein Ursprung findet an der Medialseite der Basis des Proc. zygomaticus des Squamosum statt. Er zieht medial vom Externus zum Unterkiefer. Luther sieht in ihm eine durch N. V-3 vom Externus abgespaltene Portion, da er bei Rana medial vom N. V-3 liegt und sich in seinem Verlauf, sowie inbezug auf seinem Ursprung an den Externus aufs engste anschließt. Diese Auffassung scheint aber nicht treffend $\mathrm{zu}$ sein, da die beiden im Larvenstadium ganz anders verlaufen.

3) Add. mand. post. lateralis (apla). Luther beschreibt ihn tadellos. Er entspringt an der medialen Seite und dem ventralen Rand des Quadratomaxillare. Die Fasern strahlen fächerförmig auseinander, soda $\beta$ die caudalsten schräg ventro caudalwärts, die rostralsten dagegen ventro-rostralwärts gerichtet sind. Der Ansatz findet caudal an der lateralen Fläche des Unterkiefers, am caudalen Ende des Goniale statt; seine Grenze senkt sich rostralwärts bis' zum ventralen Rand des Goniale. Der N. V-3 liegt lateral vom rostralsten Teil der Insertion.

4) Add. mand. post. longus (aplm, apll). Der kräftigste Heber des Unterkiefers entspringt bei Rana an der Dorsalseite der Labyrinthregion, ferner am Prooticum (Proo), rostral vom Occipitale. Wie Luther beschreibt, verhält er sich bei Bufo insofern abweichend, als der Muskel am Ursprung teilweise vom Squamosum überdacht wird und dadurch seinen Ursprung auf die mediale Seite dieses Knochens ausgedehnt. Dementsprechend ist seine Ursprungsfläche am Schädel kleiner und ragt nicht so weit caudalwärts wie bei Rana, sondern umfaßt nur die rostrale Fläche des Prooticum. Der Muskel zieht steil zum Unterkiefer hinab, wo er mit kurzer Sehne an der dem Meckelschen 
Knorpel zugewandten Seite und dem dorsalen Rand des Proc. coronoides des Goniale inseriert.

An der lateralen Seite dieses Muskels finden wir ferner eine vom Ursprung zum Anzatz längs verlaufende Furche, wodurch er in beide Portionen (Portio medialis $(a p l m)$ und P. lateralis $(a p l l)$ ) geteilt wird. Bei einigen Exemplaren lassen sie sich leicht voneinander unterscheiden. Wenn auch mit einiger Schwierigkeit werden sie auch am Ansatz voneinander getrennt, und zwischen ihnen zugleich etwa caudal inseriert der Articularis, wodurch sowie durch die obenerwähnte Furche sich die Trennung der beiden Portionen auch bis zum Ursprung verfolgen läßt; die erstere entspringt nämlich an der rostralen Fläche des Prooticum, die letztere dagegen zum Teil auch an der medialen Seite des Squamosum. Wị unten erwähnt wird, stammen die beiden je vom Add. mand. post. longus superficialis und profundus der Larve ab.

5) Add. mand. post. articularis $(a p a)$. Wie Luther beschreibt, ist er bei Bufo bedeutend schmäler als bei Rana; er entspringt an der Rostralseite des gegen das Gelenk gerichteten Fortsatzes des Squamosum sowie am benachbarten Teil des Quadratum und inseriert teils am knorpeligen Mandibulare medial vom höchsten Teil desselben, teils an der medialen Fläche und dem dorsalen Rand des Proc. coronoides des Goniale, und zwar nach unserem Befund caudal zwischen den beiden Portionen des Add. mand. post. long. behandelt.

6) M. pterygoideus $(m p t)$ wird in einer späteren Veröffentlichung

\section{B. Die Kaumuskulatur der Krötenlarven (Figg. 3 u. 4).}

Wie von alters her bekannt, weicht die Ausbildung der Kaumuskeln bei den Anurenlarven stark von derjenigen des erwachsenen Tieres ab. Im Gegensatz zu vertikaler Stellung der erwachsenen Muskeln, nehmen die larvalen horizontale Lage an. Luther behandelt sie sehr eingehend unter besonderer Berücksichtigung, wie die erwachsenen Kaumuskeln sich aus den larvalen entwickeln.*

Wir gehen nun in die Schilderung der larvalen Kieferadductoren von Bufo über, wobei unser Befund vom Lutherschen in einigen Einzelheiten abweicht.

Die nachfolgende Beschreibung bezieht sich auf die Larve etwa 10 Tage vor dem Durchtritt der vorderen Extremitäten, bei denen die

* Die Synonyme der larvalen Kaumuskeln sind in Luther (1914), Lubosch (1915) und Edgeworth (1935) ausfuihrlich angegeben. 


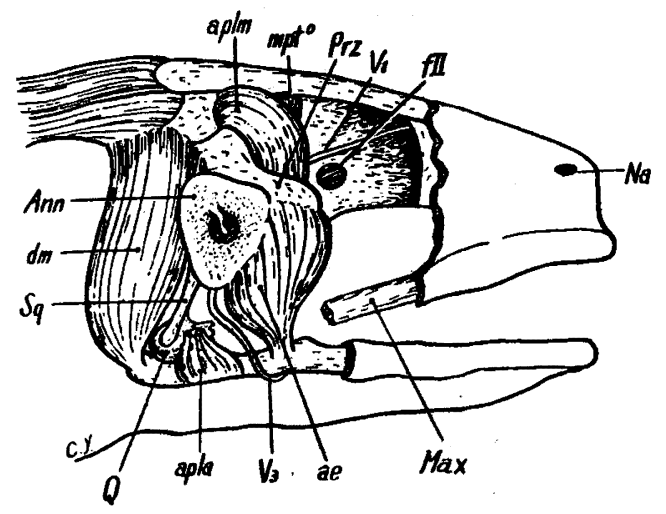

Fig. 1.

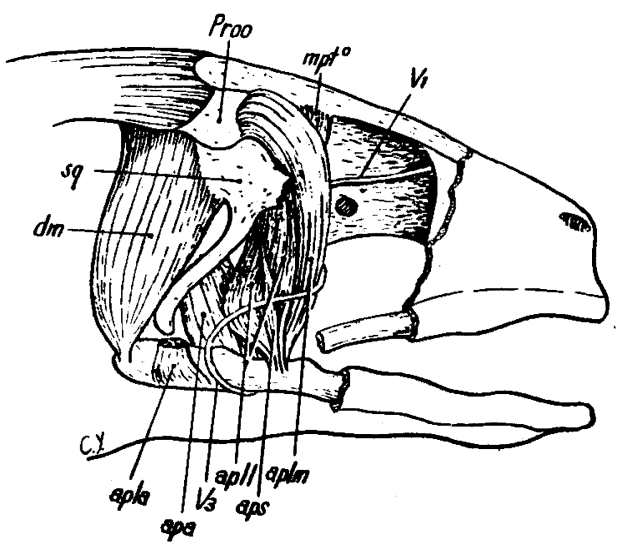

Fig. 2 .

Figg. 1, 2. Kaumuskulatur von erwachsenem Bufo vulgaris japonicus. Auge und Augenmuskeln entfernt. Fig. 2: Add. mand. externus entfernt. Vergr. 1.5: 1 .

ae. Add. mand. externus.

Ann. Annulus tympanicus. apa. Add. mand. post. articularis apla. Add. mand. post. lateralis. apll. Portio lateralis des Add. mand. post. longus.

aplm. Portio medialis desselben. aplp. Add. mand. post. longus profundus.

apls. Add. mand. post. longus superficialis.

aps. Add. mand. post. subexternus.

Ca. Capsula auditiva.

dm. M. depressor mandibulae.

fII. Foramen opticum.

ha. M. hyoangularis.

M. Cartilago Meckeli.

Max. Maxillare.

mpt. M. pterygoideus.

Na. Naris.

Umbildung des Mauls und Kieferapparats sowie die Entstehung der später zu erwähnenden, neuen Myoblasten noch nicht beginnen.

In diesem Stadium mißt die ganze Länge der sorgfältig gezüchteten Larve etwa $30 \mathrm{~mm}$. Das Wachstum der Larve ist dabei viel geringer als in vorhergehenden Stadien, so da $B$ man wohl behaupten kann, da $B$ das Wachstum in diesem Stadium fast vollständig stillsteht.

1) Add. mand. externus (M. masseter Schulzes) (ae in Figuren). Dieser, dorsal vom N. V-3 gelegene, kurze Muskel entspringt an der Medialseite des Proc. orbitalis (muscularis) (Pro) und in der Nähe der Basis desselben, dorsal vom Subexternus (aps) und in Zusammenhang mit demselben, und zieht mit einer langen dünnen Sehne des Add. mand. post. long. superf. (apls) hinweg und inseriert am larvalen Unterkiefer $(M)$.

2) Add. mand. post. subexternus (aps). Der schwache dünne 
Die Kaumuskulatnr der Anuren (Bufo vulgaris japonicus) während der Metamorphose.

Figg. 3, 4. Larvale Kaumuskulatur von Bufo vulgaris japonicus, etwa 10 Tage vor dem Durchtritt der vorderen Extremitäten. Rechtes Auge und seine Augenmukeln entfernt. Von dorsal und etwas rechts gesehen. Fig. 3: Vergr. 16:1. Fig. 4: Add. mand. externus entfernt. Vergr. $25: 1$.

O. Oculus.

oh. M. orbitohycideus.

Proo. Prooticum.

Pra. Proc. articularis quadrati.

Prm. Froc. metapterygoideus quadrati.

Pro. Proc. orbitalis (muscularis) quadrati.

Prz. Proc. zygomaticus.

Q. Quadratum.

sa. M. suspensorioangularis.

sh. M. suspensoriohyoideus.

Sq. Squamosum

(Tympanicum Gaupps).

Sr. Suprarostrale. $V_{1}$. N. ophthalmicus. $\mathbf{v}_{2}$. N. maxillaris.

$\mathbf{V}_{\ddots}$. N. mandibularis.

Muskel entspringt eng ventral vom Ursprung des Externus. Augenscheinlich gehören also die beiden Ur. sprünge eng $z u-$ sammen. Er zieht, den Externus

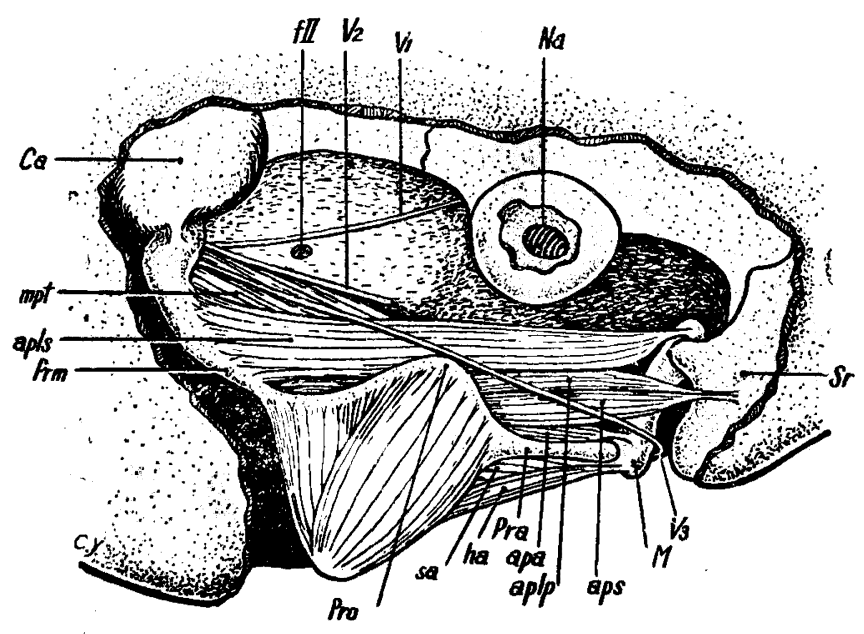

Fig. 4.

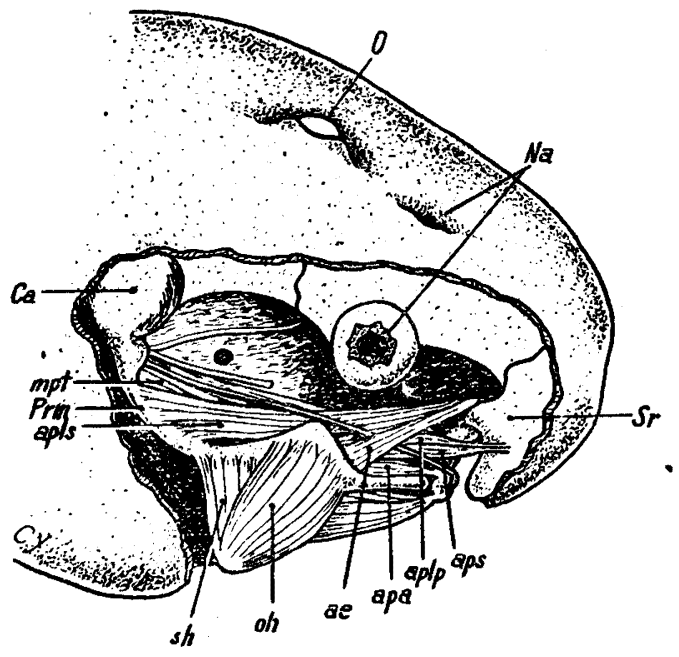

Fig. 3.

unter spitzem Winkel kreuzend, ventral vom N. V-3, direkt dorsal vom Longus prof., gegen die Sehne des letzteren, an der er inseriert. Dabei lassen sich die Sehnenfasern in die beiden Sehnen dieser Muskeln verfolgen. Lupenpräparierung dieses Muskels ist schwierig, aber mit gleichzeitiger Benutzung der Serienschnitte wird sie leichter.

Nach Luther fehlt der Subexternus bei Bufolarven, und der Externus zerfällt hier in zwei getrennte dünne Muskelchen; das dorsale 
von diesen beiden (Add. mand. externus dorsalis, Luther) zieht mit einer langen, dünnen Sehne zur Sehne des Longus superf., das ventrale (Add. mand. ext. ventralis, Luther) dagegen, das dorsale unter spitzem Winkel kreuzend, zur Sehne des Longus prof. Luther hat wahrscheinlich den Subexternus mit Externus ventralis verwechselt.

3) Add. mand. post. lateralis (apla). Luther beschreibt, daß der larvale Lateralis bei Bufo mit dem gleichnamigen Muskel des erwachsenen Tieres übereinstimmt, indem die Insertion direkt dorsal am articularen Teil des Mandibulare erfolgt, und gibt eine Abbildung davon. Lubosch (1915) stellt ein Fragezeichen hinter diesen Muskel - in seiner tabellarischen Übersicht über der Kaumuskeln der Anurenlarven.

Wir haben diesen Muskel an der Bufolarve im oben genannten Stadium vergebens gesucht, konnten ihn aber an der Larve kurz vor dem Durchtritt der vorderen Extremitäten in der von Luther gesprochenen Lage finden. Wie unten erwähnt wird, entwickelt er anders als die übrigen Kaumuskeln.

4) Add. mand. post. longus superficialis (M. temporalis Schulzes) (apls). Der oberflächlichste der den Boden der Orbita bildenden drei Muskeln entspringt an der Dorsalseite des Proc. metapterygoideus (Prm) des Quadratum $(Q)$, und zwar nur auf dieselbe beschränkt, zieht rostral und etwas medialwärts und geht in eine starke Sehne über, welche mit der Sehne des Externus an der lateralen (vorderen) Fläche des Mandibulare inseriert, wie Goette, Schulze und Luther richtig beschreiben.

4') Add. mand. post. long. profundus (M. subtemporalis Schulzes) $(a p l p)$. Der Muskel, fast in seiner ganzen Ausdehnung vom vorigen bedeckt, entspringt rostral vom Ursprung des vorigen am Proc. metapterygoieus. Die Fasern konvergieren rostralwärts gegen eine Sehne, die sich zum Teil direkt an das Mandibulare ansetzt, größtenteils dagegen oberflächlich über diese Stelle hinweg zum Suprarostrale $(S r)$ zieht; der Subexternus (aps) inseriert dagegen, den Longus prof. zum Teil bedeckend, an der Oberfläche der Sehne des letzteren. Der Longus prof. kreuzt dabei hier den Longus superf. unter spitzem Winkel.

5) Add. mand. post. articularis $(a p a)$. Wie Luther beschreibt, entspringt dieser kleine, annähernd parallelfaserige Muskel an der Medialseite des rostralen Randes der Basis des Proc. orbitalis, und inseriert an der Dorsalseite des Mandibulare in der Nähe dessen articularen Endes, dicht symphysial von der Insertion des M. pterygoideus. Sein Ursprung liegt ventral von dem des Subexternus. 
Die Kaumuskulatur der Anuren (Bufo vulgaris japonicus) während der Metamorphose. 9

6) M. pterygoideus $(m p t)$ wird in einer späteren Schrift behandelt.

\section{Umgestaltungen während der Metamorphose.}

Unser durch Mikrodissektion gewonnener Befund über die Veränderung der Kaumuskulatur während der Metamorphose wird in Figuren 5-10 wiedergegeben. Während der Metamorphose erfolgen am Skelett die Resorption des Proc. orbitalis, die Erweichung, Zusammenschiebung und Auflösung des caudalen Teils des Quadratum, die Aufrichtung des übrigen Teils des letzteren, die Verlängerung des Meckelschen Knorpels,-alle jene besonders von Gaupp (1893) eingehend geschilderten Vorgänge, die den larvalen Kieferapparat in den definitiven führen. Entsprechend diesen Veränderungen am Skelett „müssen natürlich die Kaumuskeln sich (wieder) steil aufrichten ", wie Goette schon in 1875 beschrieben hat. Die seit Goette von Gaupp (1893), Edgeworth (1911, 1935), Lubosch $(1914,1915)$ und besonders von Luther (1914) studierten Ergebnisse faßt Lubosch (1938) in seiner Handbuchbeitrag in einen Satz zusammen; , Wenn während der Metamorphose das Kiefergelenk nach rückwärts verlegt wird, nehmen die Muskeln (Kaumuskeln) vertikale Lage an und gewinnen ihre endgültigen Ursprünge und Ansätze". Wir konnten dies auch unter Lupe im großen und ganzen bestätigen.

Zuerst sei das Verhalten des Add. mand. post. long. superf. während der Metamorphose durch Lupenpräparation und Schnittuntersuchung näher verfolgt, nicht aus dem Grunde, da $\beta$ dieser Muskel besonders dafür geeignet ist, sondern als ein Beispiel der Muskelumwandlung.

\section{A. Umgestaltung des Add. mand. post. long. superf. während der Metamorphose (apls in Figg. 5-10).}

Wenn die vorderen Extremitäten durchtreten, verschiebt sich der Proc. metapterygoideus (Prm), an dem der betreffende Muskel entspringt, dorsalwärts, und krümmt sich zickzack in Ziehharmonikaform, wie Katsumata (1938) und Kawai (1942) angeben (Fig. 6). In diesem Stadium sind die Ohrkapsel und das Quadratum gelenkig verbunden, so daß man das Quadratum mit Pinzette gegen die Ohrkapsel $(\mathrm{Ca})$ hin und her bewegen kann. Der Proc. metapterygoideus steckt dann mit dem Ursprung des Add. mand. post. superf. unter die Ohrkapsel, während der Unterkiefer $(M)$ mit dem Muskelansatz sich caudalwärts verlängert. 
Entsprechend dieser Skelettverschiebung steht jetzt der Longus superf. schief, der vorher horizontale Lage angenommen hat, und zwar desto steiler, je weiter die Metamorphose vor sich geht. Ferner erscheint er gelblich, und verlegt seinen Ursprung von dem sich aufflösenden Proc. metapterygoideus auf die Ohrkapsel (Fig. 8). Der Muskel, der vorhin gelblich aussah, erscheint nunmehr wieder weißlich ;

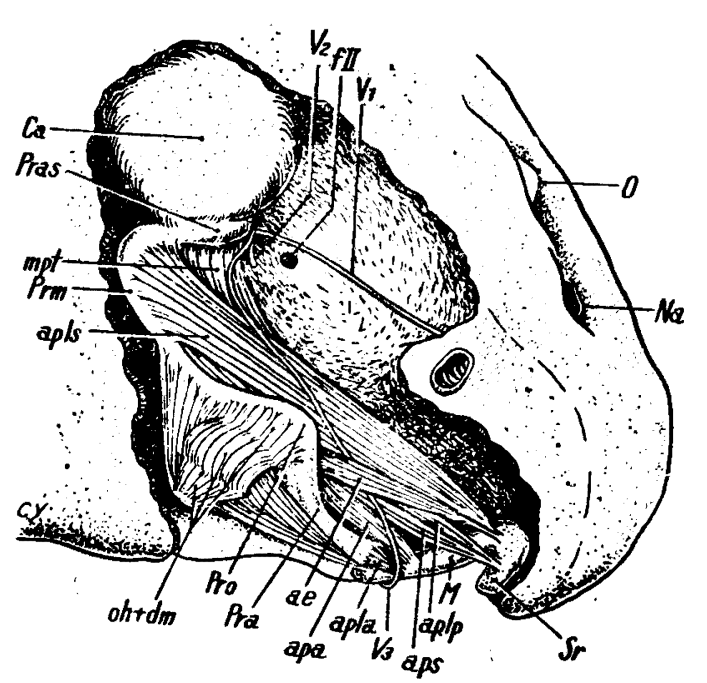

Fig. 5.

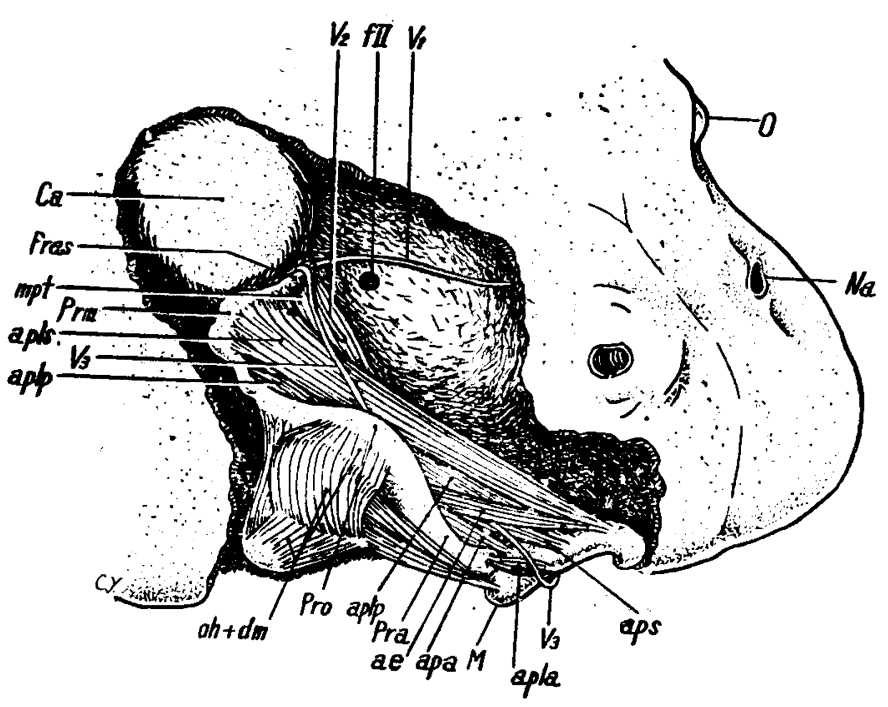

Fig. 6 .

\section{Erklärung. der} 5.-10. Figur.

Serienweise Darstellung der Veränderungsvorgänge der Kaumuskulatur während der Metamorphose bei Bufo vulgaris japnonicus. Rechtes Auge und seine Augenmuskeln entfernt. Fig. 5: schräg von dorsal, rechts und etwas cranial gesehen; mit fortschreitender Figurnummer verschiebt sich die Ansichtsrichtung nach rechts. Vergr. $18: 1$.

Fig. 5: am ersten Tag des Extremitätendurchtrittes. Fig. 6: am 2. Tag. Fig. 7 : am 3.-4. Tag. Die alten, sich degenerierenden Muskelfasern nur im Add. mand. ext., A. m. p. superf. und Pterygoideus punktiert gezeichnet. Fig. 8: am 5. Tag. Fig. 9: am 6.-7. Tag (Endstadium der Metamorphose). Fig. 10: g'eich wie Fig. 9. A. m. ext. und A. m. p. lat. entfernt. Vergr. 25:1

ae. Add. mand. externus, Ann. Annulus tympanicus, apa. Add. mand. post. articularis, apla. Add. mand. post. 
Die Kaumuskulatur der Anuren (Bufo vulgaris japonicus) während der Metamorphose.

das Quadratum ist mit der Ohrkapsel schon unverschieblich verbunden. Unter besonderer Berücksichtigung der oben angegebenen Veränderungen sind die mikroskopischen Ergebnisse in Fig. 11 schematisch und serienweise dargestellt. Für diese Beobachtungen sind die Sagittalschnitte besonders vorteilhaft.

\section{1) Die Entstehung der neuen Myoblasten.}

Wenn man die Schnitte des mit Thionin gefärbten Add. mand. post. long. superf. der Bufolarve kurz vor dem Durchtritt der vorderen Extremitäten durchmustert, so findet man schon junge Muskelfasern

lateralis, aplp. Add. mand. post. longus profundus, apls. Add. mand. post. longus superficialis, aps. Add. mand. post. subexternus, Ca. Capsula auditiva, dm. M. depressor mandibulae, flI. Foramen opticum M. Cartilago Meckeli, Max. Maxillare, mpt. M. pterygoideus Na. Naris, 0. Oculus, oh. M. orbitohyoideus, Pra. Proc. articularis quadrati, Pras. Proc. ascendens quadrati, Prm. Proc. metapterygoideus quadrati, Pro. Proc. orbitalis (muscularis) quadrati, Q. Quadratum, Sr. Suprarostrale, $\mathbf{V}_{1}$. N. ophthalmicus, $\mathbf{V}_{2}$. N. maxillaris, $\mathbf{V}_{3} \cdot \mathrm{N}$. mandibularis.

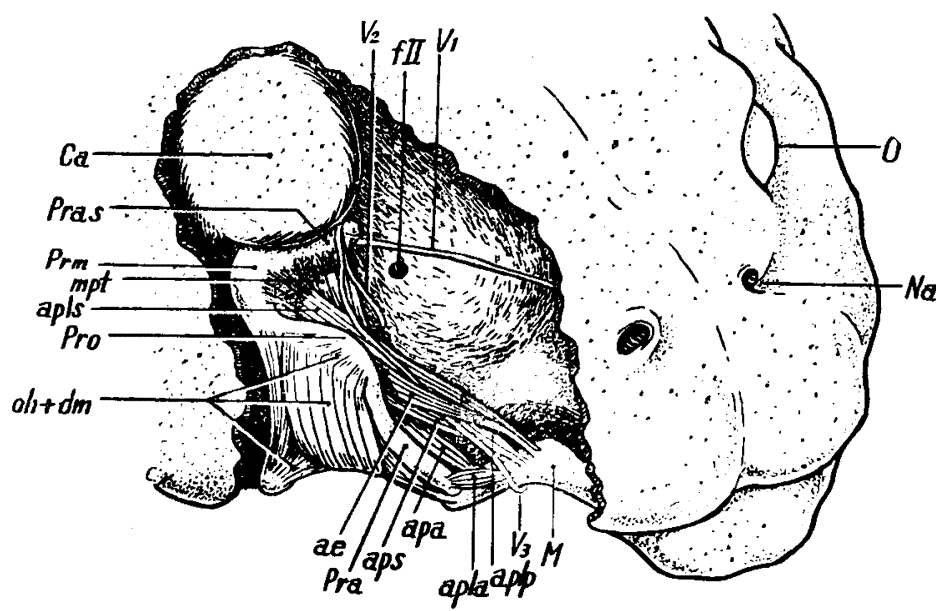

Fig. 7.

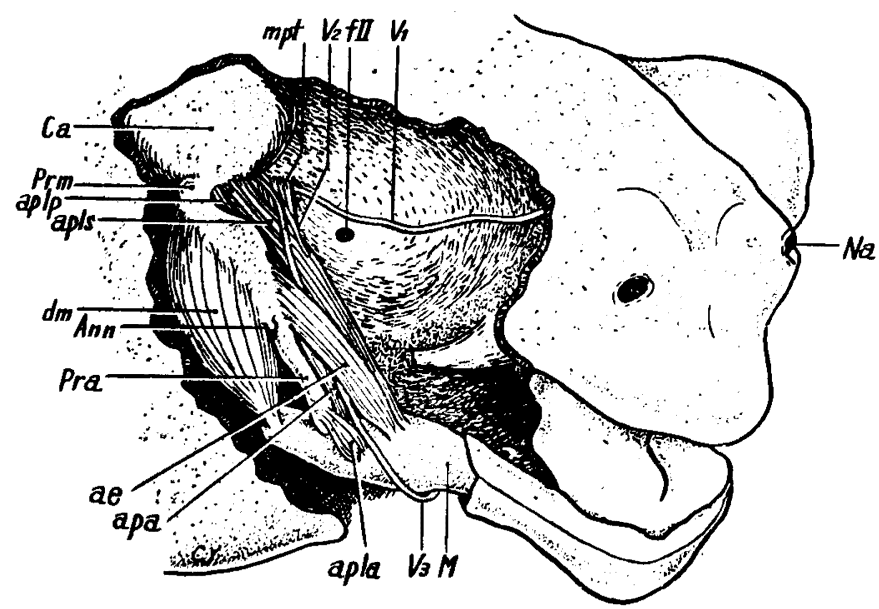

Fig. 8. 
zahlreich im mittleren Teil des genannten Muskels, wiewohl die bei der Metamorphose stattfindenden Veränderungen des Knorpels und der Muskeln durch Lupenuntersuchung noch nicht bemerkbar sind. Das Sarkoplasma und die Kernkörperchen dieser jungen Muskelfasern sind mit Thionin tief violett angefärbt, während die Myofibrillen und das Kernchromatin mehr oder weniger blau tingiert sind. Die jungen Muskelfasern sehen besonders deutlich metachromatisch aus, die alten dagegen nicht, wodurch sich die beiden Faserarten mit einem Blick leicht voneinander unterscheiden lassen. Bei der Larve etwa 10 Tage

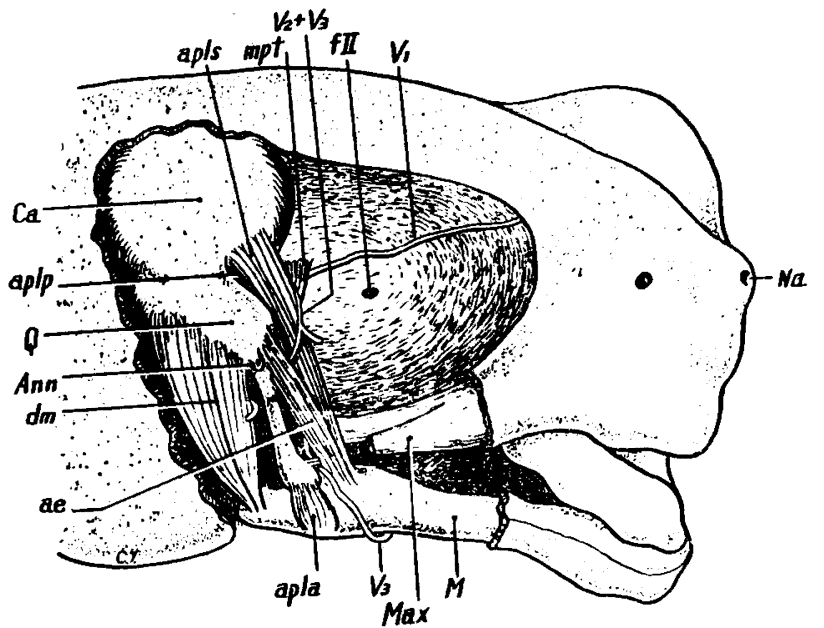

Fig, 9.

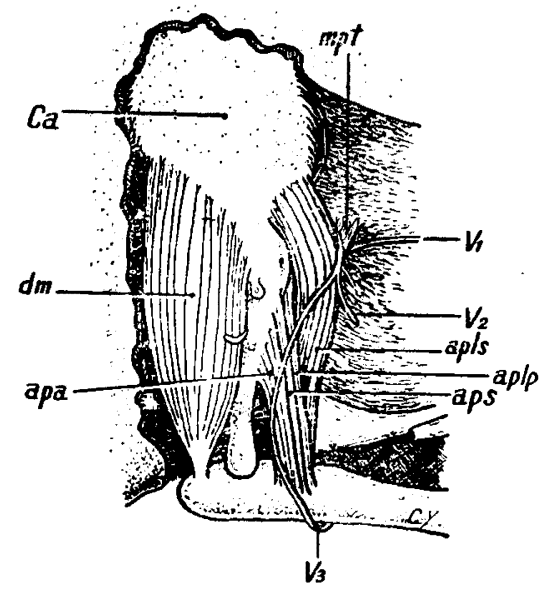

Fig. 10. vor dem Durchtritt der vorderen Extremitäten können aber solche jungen Fasern im betreffenden Muskel noch nicht erkannt werden.

Mit dieser Färbemethode konnten wir die Herkunft der die jungen Muskelfasern liefernden Myoblasten von den Fibroblasten im Endomysium dieses Muskels mit Sicherheit verfolgen, wie wir schon am Depressor mandibulae ( $d m$ in Figuren) im Laufe der Metamorphose konstatieren konnten, wobei wir einige zytologische Details über die neuen Myoblasten, die wir damals vorläufig Metamyoblasten nannten, geschildert haben. a) Die basophile, mit Thion- 
Die Kaumuskulatur der Anuren (Bufo vulgaris japonicus) während der Metamorphose. 13 in metachromatisch färbbare Substanz im Zelleib der Myoblasten scheint mit der Entstehung der Myofibrillen in näherem Znsammenhang zu stehen. b) Das Glykogen, das im Zytoplasma der jüngsten Myoblasten ziemlich reichlich vorhanden ist, wird mit fortschreitender Entwicklung der letzteren geringer. c) Die Mitosen sind im Stadium, wo die Myoblasten erscheinen, nur in geringer Zahl beobachtet; sonst sind ausschließlich die Amitosen allein vorgefunden.

Die neuen Myoblasten im Adductor weichen aber in ihrem zeitlichen und örtlichen Verhalten nur wenig von denen im Deprossor mandibulae $(d m)$ ab. In diesem Muskel erscheinen sie nämlich später als im Depressor. Die jüngsten neuen Myoblasten erscheinen im Orbitohyoideus $(o h)$ schon 7 Tage vor dem Extremitätendurchtritt, im Adductor dagegen 2 oder 3 Tage vor demselben. Wie wir später zurückkommen werden, erscheinen die neuen Myoblasten in einzelnen Adductor nicht gleichzeitig, sondern nacheinander.

Wie in der vorhergehenden Abhandlung erwähnt, treten diese Myoblasten, die sich eng an das Sarkolemma der alten Muskelfasern anheften, im Orbitohyoideus zunächst im lateralen Teil des Muskelbauches auf, im Adductor mand. post. long. superf. dagegen überall im Muskelbauche ( $a$ in Fig. 11).

Hand in Hand mit der Neubildung der Muskelfasern schreitet die Degeneration der alten Fasern vom mittleren Teil (Muskelbauch) des Muskels kopf- sowie schwanzwärts fort (s.u.). Gleichzeitig wachsen auch die neuen Fasern kopf- sowie schwanzwärts.

Etwas 10 Tage vor dem Extremitätendurchtritt, wo die neuen Myoblasten noch nicht erkennbar sind, ist das Endomysium sehr gering und zellenarm. Ob diese Zellen feine Fasern haben, ist zweifelhaft; wahrscheinlich handelt es sich um Mesenchymzellen zwischen Muskelfasern. $\mathrm{Daß}$ diese Mesenchymzellen zwischen den Fasern sich alle zu neuen Myoblasten umformen, ist undenkbar; während der Rückbildung der alten Fasern wandeln sie sich wenigstens am Ansatz sowie am Ursprung des Muskels sicher nicht zu Myoblasten um, obwohl wir noch nicht wissen, ob sie da unverändert bleiben oder verschwinden. Ebenso ist unklar, ob alle Mesenchymzellen im mittleren Teil des Muskels sich in Myoblasten umwandeln, aber soviel ist wahrscheinlich, daß sie in geringerer Zahl als echte Fibroblasten unverändert bleiben können, denn zwischen den ziemlich entwickelten neuen Muskelfasern werden noch Fibroblasten aufgefunden, wiewohl ihre Herkunft unklar ist. Daß eine Mesenchymzelle mehrere Muskelfasern liefern kann, ist wohl denkbar, weil ihre Zahl vor der Metamorphose viel weniger ist als die der neuen 


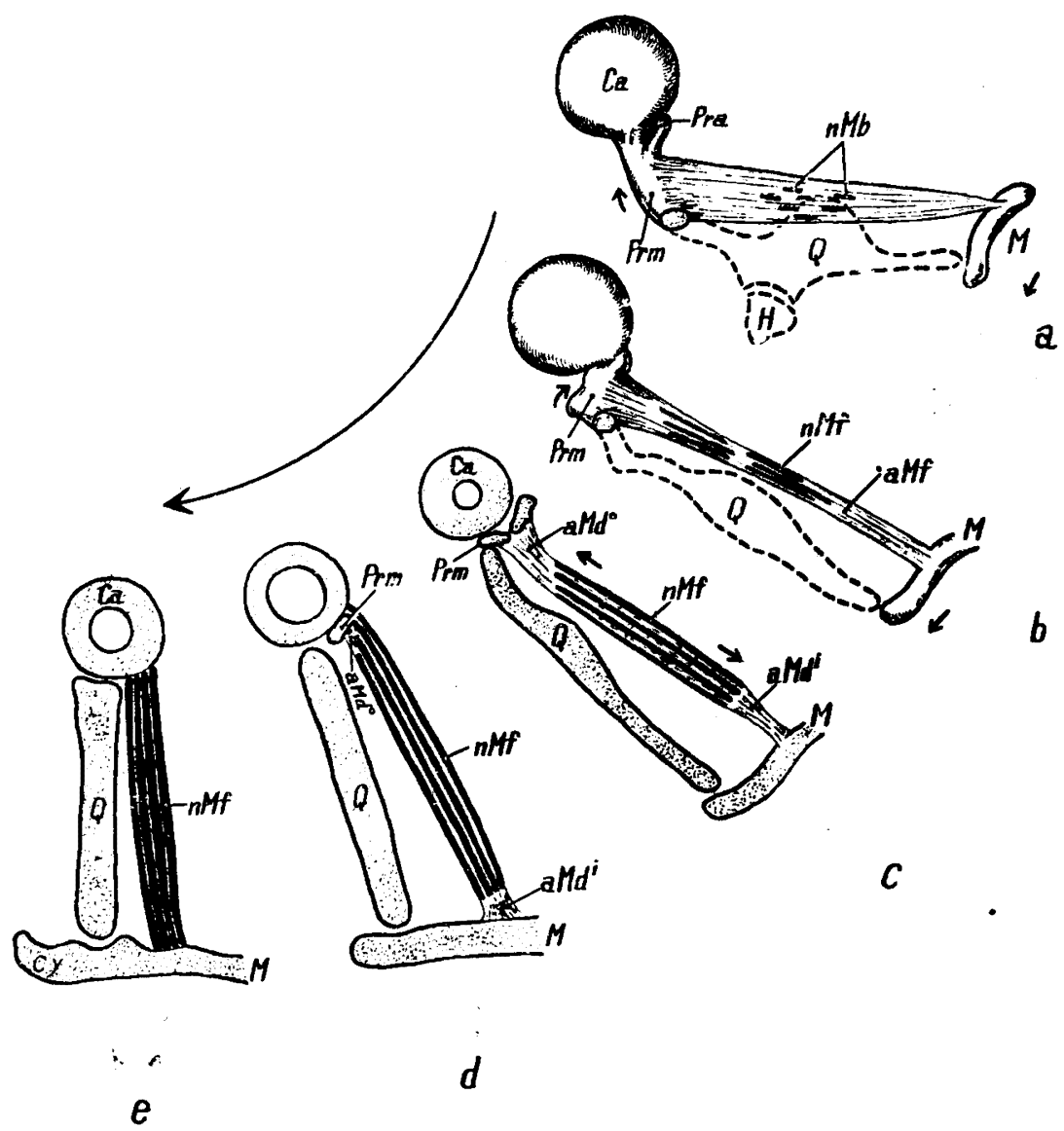

Fig. 11. Schematische Darstellung der Erneuerungsvorgänge des Adductor mandibulae posterior longus superf. (=larvaler „M. temporalis“ F. E. Schulzes, ein Teil des erwachsenen „M. temporalis “ Gaupps) während der Metamorphose. Ansicht von rechts.

a : am ersten Tag des Extremitätendurchtrittes; b : am 2. Tag; c: am 3.-4. Tag; d: am 5. Tag; e: am 6.-7. Tag. (Vergl. Fig. 5-10)

aMf, alte Muskelfasern; aMd, alte Muskelfasern, die sich degenerieren, und dann verschwinden; Ca, Capsula auditiva; H, Hyale; M, Mandibularknorpel, der kaudalwärts sich verlängert; nMb, neue Myoblasten, die von Fibroblasten abstammen und zwischen den alten Muskelfasern auftreten; nMf, neue Muskelfasern, die sich von den neuen Myoblasten entwickeln, und entlang der sich degenerierenden alten Fasern muskelkopf* und -schwanzwärts wachsen; Pra, Proc. ascendens quadrati; Prm, Proc. metapterygoideus quadrati, der sich verkürzt. dann sich unter die Ohrkapsel streckt, endlich sich auflöst; $\mathbf{Q}$, Quadratum, das während der Metamorphose sich aufrichtet.

Myoblasten während der Metamorphose. Die jüngsten, noch indifferenten Zellen zeigen Kernteilungen. Die wahrscheinlich durch Mitosen 
Die Kaumuskulatur der Anuren (Bufo vulgaris japonicus) während der Metamorphose. 15

gebildeten Tochterzellen wandeln sich vielleicht $z$. T. $z u$ den neuen Myoblasten, z. T. aber auch zu den Fibroblasten um.

2) Die Verschiebung der neuen Muskelfasern.

Durch Mikrodissektion an der in Formol fixierten Larve kurz nach dem Extremitätendurchtritt, bemerkt man am Adductor, da $\beta$ der Muskelbauch weißlich, die übrigen Teile (Kopf und Schwanz) dagegen gelblich aussehen. Dieser weißliche Teil besteht hauptsächlich aus den neuen Muskelfasern, die mit Thionin stark metachromatisch angefärbt werden. Die alten beginnen schon zu degenerieren. Wenn man die in toto mit Hämatoxylin (Hansen) gefärbte Larve präpariert, so findet man den weißlichen Teil tiefer blau angefärbt als die gelblichen, wodurch man die beiden Teile deutlicher als sonst voneinander unterscheiden kann, und je steiler sich der Muskel aufrichtet, desto mehr verlängert sich der mittlere weißliche Teil, verkürzen sich dagegen die beiden gelblichen Enden (Fig. 7, $C$ in Fig. 11).

Mit der Verlängerung der neuen Fasern vermẹhren sie die Myofibrillen obne Querstreifung, und auch die Kerne durch amitotische Teilung. Zahlreiche stäbchenförmige Mitochondrien werden um die Kerne aufgefunden.

Im folgenden Stadium wird die Metachromasie der Muskelfasern im mittleren Teil schwächer, während sie an den beiden abgerundeten Enden, wo die Amitosen noch aufgefunden werden, noch stärker bleibt. Etwas später erscheinen die Querstreifungen an den Myofibrillen der neuen Fasern.

Die alten Muskelfasern des Adductors zeigen im Kopfe und Schwanze schon ziemlich fortgeschrittene Degeneration, so daß sie bindegewebeartige Stränge $\left(a M d^{\circ}, a M d^{i}\right.$ in Fig. 11) darstellen, und verlegt seine Insertion, wie nachher näher erörtert wird, an einer Stelle des Unterkiefers, die von seinem larvalen Ansatz nur wenig entfernt ist, indem die Sehne rückbildet; entlang dieser Stränge wachsen nun die neuen Fasern muskelkopf- sowie -schwanzwärts.

Am dorsalen Muskelende erreichen die neuen Fasern den sich auflösenden Proc. metapterygoideus (Prm in Fig. 11d), der sich schon unter die Ohrkapsel hinstreckte, und dann entlang dieses Fortsatzes die Ohrkapsel.

Diese Veränderungen beschreibt Luther an der Larve von Pelobates fuscus, der sie nur auf präparatorischem Weg untersuchte, wie folgt; , Durch diese dorsalwärts und caudal gerichtete Verschiebung (des Proc. metapterygoideus und des Proc. ascendens) wird der Ursprung 
der Muskeln (Mm. adductores mandibulae posteriores longi superf. et prof.) der Regio prootica genähert, und es ist verständlich, daß mit der völligen Resorption des Proc. ascendens und des Proc. metapterygoideus der Ursprung auf die Labyrinthkapsel überwandert “. Diese ungenügende Beschreibung dürfte wohl auf seine beschränkte Methode beruhen.

Am ventralen Muskelende wachsen die neuen Fasern entlang des ventralen bindegewebeartigen Stranges ventralwärts und erreichen den Unterkieferknorpel, der sich schon caudalwärts verlängert hat.

So wird der larvale Adductor von den neuen Fasern bedeckt, die Lage der Portio medialis des Add. mand. post. longus des erwachsenen Bufo einnehmen, wobei der neue Adductor kürzer als der larvale wird (Figg. 9, 10, $e$ in Fig. 11).

Gegen Ende der Metamorphose stellen die neuen Fasern ähnlichen Zustand wie die Purkinjeschen Fasern dar, indem die Kerne sich in der Achse der Fasern, und die Fibrillen sich um die Kerne anordnen. Schon in diesem Stadium wird die Metachromasie des Sarkoplasma schwächer, und die Menge des Glykogens bedeutend geringer. Weiter bis zur Vollendung der Muskelfasern konnten wir den Vorgang nicht vorfolgen, weil die Züchtung der Kröten nach dem Metamorphose bekanntlich kaum möglich ist.

3) Die Muskelfasern im Larvenstadium und ihre Degeneration.

Bekanntlich sind die Muskelfasern der Larve morphologisch von den des erwachsenen Tieres verschieden (z. B. Maurer, 1915, S. 380). Die dorsale Rumpfmuskulatur (Müller, 1921) nebst mit der Schwanzmuskulatur, der M. levator hyoidei (Takisawa u. Sunaga, 1951) und die Kaumuskulatur (s.u.) der Larven gehen sogar alle während der Metamorphose zugrunde. Es scheint aber uns zweifelhaft, ob man dabei auf einer Entwicklung der Fasern des Larvenstadiums zu denen der Anurenjunge rechnen könnte. Das ist wahrscheinlich auch der Fall an allen Muskulaturen der Kröte, worauf wir in späteren Abhandlungen zurückkommen wollen. Wir glauben nämlich, daß die Muskelfasern der Larve als solche die fertigen sind.

Vor allem sind die Fasern der Larve im Vergleich mit denen des erwachsenen Tieres bedeutend reich an Sarkoplasma, so da $\beta$ das letztere auf dem Querschnitt der Fasern ausgedehnteres Feld einnimmt, als die Myofibrillen. In diesem reichlichen Sarkoplasma sind die stäbchenund fadenförmigen Mitochondrien aufgefunden, welche sich besonders 
Die Kaumuskulatur der Anuren (Bufo vulgaris japonicus) während der Metamorphose. 17 nahe am Sarkolemma und den Fibrillen, und um der Kerne dicht ansammeln.

Das Pigment sind auch im Sarkoplasma vorgefunden. Es wird durch Osmium schwarz tingiert, was besagt, da $B$ es von Lipofuscinnatur ist. Bemerkenswert ist, daß dieses sogen. Abnutzungspigment schon bei der Larve vor dem Extremitätendurchtritt aufgefunden wird.

Die basophile Substanz im Sarkoplasma ist durch einfache ThioninFärbung ziemlich gut nachweisbar, besonders deutlich am FormolAlkohol fixierten Material. Das Glykogen wird wie in quergestreiften Muskelfasern des erwachsenen Tieres nur spärlich nachgewiesen.

Die rundlichen oder ovalen Kerne liegen in der Gegenseite der Myofibrillen. In diesem und späteren Stadien sind schon die Kernteilungen, mitotischen sowie amitotischen, nicht beobachtet.

Kurz nach dem Auftritt der neuen Myoblasten zwischen den larvalen Muskelfasern, treten in den letzteren die Degenerationserscheinungen auf. Im Sarkoplasma vermehrt sich das Pigment; die basophile Substanz geht allmählich verloren, das Kernkörperchen ist dagegen $\mathbf{z u}$ dieser Zeit noch metachromatisch färbbar. Die Myofibrillen werden trüb und schwer färbbar, dann hie und da zerrissen, wellenförmig, und endlich zerstückelt in zahlreiche Stückchen. Die Muskelfasern teilen sich dementsprechend quer in großer Zahl. Ob die durch Querteilung der Muskelfasern entstandenen „Sarkolyten “, wie Mayer (1886) und Schaffer (1893) bei der Degeneration der Schwanzmuskulatur der Anuren beschrieben, eventuell in amöboide Zellen übergehen können, konnten wir nicht feststellen. Nach unserer Beobachtung verliert das Kernkörperchen seine metachromatische Natur, und der Kern ist zur Pyknose geneigt, dann wird karyolytisch oder vakuolisierend, um endlich $\mathrm{zu}$ verschwinden.

Wie schon bei der Entwicklung des M. depressor mandibulae beschrieben, wird das Glykogen in den sich degenerierenden Fasern reichlicher: die sogen. Glykogendegeneration oder -infiltration. Mit fortschreitender Degeneration wird es wieder geringer. Solche Erscheinnng bei der Degeneration ist auch in den übrigen larvalen Muskeln beobachtet.

Anderseits findet man das Glykogen als feine Tröpfchen in reichlicher Zahl im Gallertgewebe der Anurenlarven (Takisawa u. Kanō, 1951), es ordnet sich an der Oberfläche der Zellen und der Fasern des betreffenden Gewebes an. In fortgeschrittenem Stadium der Metamorphose verschwinden diese Glykogentröpfchen zunächst, dann auch das 
Gallertgewebe selbst. Das Glykogengehalt der Leberzellen verändert sich während der Metamorphose dagegen kaum.

Während der Metamorphose nehmen die Larven kaum die Nahrung auf ; die mächtige Umbildung soll ohne Zweifel eine große Energiequelle in Anspruch nehmen, wobei m. E. sicherlich das Glykogen im Gallertgewebe sowie in den sich degenerierenden Muskelfasern als Energiequelle aufgezehrt wird.

Dieser Degenerationsprozeß schreitet mit dem Wachstum der neuen Fasern Hand in Hand fort. Zunächst beginnt der Vorgang im Muskelbauch des Adductors und geht nach beiden Muskelenden vor sich ( $b$, $c, d$ in Fig. 11).

Im Adductor konnten wir die Entwicklung der neuen Fasern und die Rückbildung der alten bis zum Abschluß der Metamorphose von Schnitt zu Schnitt lückenlos verfolgen. So gehen alle Fasern des larvalen Adductors zweifellos zugrunde und machen den neuen Platz, bis ein ganz neuer Muskel an Stelle des alten entsteht.

4) Die Degeneration der Sehne.

Wie schon beschrieben, zieht die Sehne des Add. mand. post. long. superf. der Larve über die caudo-dorsale Fläche des Mandibulare hinüber, und inseriert an der latero-rostralen Fläche des medio-rostralen Teils desselben. Mit Eintritt der Metamorphose, wie Luther bei Pelobates beschreibt, scheint diese Sehne auch bei Bufo sich der dorsalen Fläche des Mandibulare anzuheften, obwohl die ursprüngliche Sehne sich bis zum ursprünglichen Insertionspunkt verfolgen läßt. Sie zeigt aber schon in dieser Zeit Degenerationsbild; sie quillt sich und wird weich. Mikroskopisch vermehrt sich das Pigment in den Zellen, die Fasern werden schwer färbbar, und die Kerne pyknotisch. In etwas späteren Stadien verschwindet die Sehne vollkommen.

Dieser Degenerationsvorgang, nämlich die anfängliche Anheftung am Mandibulare mit Quellung, dann die Rückbildung, findet nach Luther an den Insertionen der Adductoren mit Ausnahme des Pterygoideus und Articularis statt. Ferner beschreibt Luther in Hinsicht auf die Ursache der Degeneration wie folgt: „An dem abgebildeten und ähnlichen Präparaten macht es nun den Eindruck, als hätte die Buccalfalte die Sehnen und die Enden der Muskelfasern des A.m. p. longus gegen das Mandibulare gedrückt.-Selbstredend wird man sich den Einfluß der vorwachsenden Buccalfalte nicht grobmechanisch vorstellen dürfen. $\mathrm{DaB}$ ihr Vorhandensein aber in naher Beziehung $z \mathrm{u}$ 
Die Kaumuskulatur der Anuren (Bufo vulgaris japonicus) während der Metamorphose. 19 der Verlagerung der Insertion steht, wird man schwerlich bezweifeln können."

Nach unserer Untersuchung findet dieser Degenerationsvorgang auch in der Sehne des Pterygoideus statt, deren Insertion zur Buccalfalte nicht in naher Beziehung zu stehen scheint. (Der Articularis hat keine Sehne.) Demgemäß scheint uns die Luthersche Auffassung nicht treffend zu sein; wir meinen vielmehr, daß die Auflösung von den Sehnen selbst ausgeht.

\section{B. Das Verhalten der übrigen Muskeln außer dem \\ Add. mand. post. lateralis.}

Wir haben bei der Umgestaltung des Longus superf. bestätigt: (a) Mit dem Eintritt der Metamorphose erscheinen im mittleren Teil des larvalen Muskels neuen Myoblasten, die von Fibroblasten des Endomysiums abstammen. (b) Im Laufe der Metamorphose wachsen die neuen Muskelfasern aus den neuen Myoblasten gegen die beiden Enden des larvalen Muskels fort, um die neuen Insertionen zu erreichen; (c) alle alten Fasern dagegen gehen zugrunde. Diese Erneuerungsvorgänge des Muskels finden auch bei den übrigen Muskeln mit Ausnahme des Add. mand. post. lateralis statt, obwohl sie in einigen Einzelheiten von denjenigen der Longus superf. abweichen.

1) Am Add. mand. post. longus profundus. (aplp in Figg. 5-10).

In diesem Muskel erscheinen die neuen Myoblasten im Muskelbauch gleichzeitig mit dem vorigen Muskel. Die neugebildeten Muskelfasern wachsen gegen den Muskelschwanz und -kopf entlang der sich auflösenden Fasern. Sobald die neuen Fasern heranwachsen, beginnen die beiden Longi von den mittleren Teilen her zu verschmelzen. Zum Beispiel sehen die beiden Muskeln am 3. Tag nach dem Extremitätendurchtritt unter Lupe gegabelt aus; die mittleren, aus den neuen Fasern bestehenden, weißlichen Teile sind schon verschmolzen, während ihre muskelschwanzwärt gerichteten, noch voneinander getrennten Enden, sowie ihre muskelkopfwärts gerichteten Enden gelblich aussehen. Am Ende der Metamorphose verschmelzen die beiden Longi zu einer einheitlichen Muskelmasse, können aber an einem soeben metamorphosierten Exemplar die beiden Portionen noch als solche mikroskopisch erkannt werden.

Am 3. Tag nach dem Extremitätendurchtritt liegt der Proc. metapterygoideus nebst dem Ursprung des Longus prof. bereits unter der Ohrkapsel (s.o.); anderseits verlängert sich das Mandibulare mit der 
A. Takisawa, Y. Ōhara und K. Kanö.

Teilinsertion der Muskelsehne caudalwärts, während das Suprarostrale, an welchem der Hauptteil der Sehne inseriert, sich rückbildet. Mit der fortschreitenden Metamorphose richtet sich auch dieser Muskel steil auf.

Am dorsalen Muskelende erreichen währenddessen die neuen Fasern, wie am vorigen Muskel, entlang der sich degenerierenden Fasern muskelkopfwärts den sich auflösenden Proc. metapterygoideus und die Basis desselben, und zum Teil ferner entlang dieses Fortsatzes die Ohrkapsel direkt lateral und unten vom neuen Ursprung des Longus superf., während sie zum Teil an der medio-rostralen Fläche des dorsalen Endes des Quadratum stehen bleiben.

Mit dem Eintritt der Metamorphose haftet am ventralen Muskelende, wie Luther beschreibt, diejenige Stelle, wo der Longus prof. der Larve in seine Sehne übergeht, dem Mandibulare an, wobei die ursprüng- . liche Sehne sich bis zum Suprarostrale ( $S r$ in Figg. 5,6 ) verfolgen läßt. Wenn das Suprarostrale zugrunde geht, setzt er sich nur an das Mandibulare an, indem seiner Sehnenteil sich degeneriert, und endlich verschwindet. Hier können wir auch die Luthersche Auffassung über die Ursache der Sehnendegeneration nicht unterstützen. Die neuen Muskelfasern wachsen entlang der sich degenerierenden alten Fasern, um das Mandibulare, an welchem der Muskelschwanz anhaftet, zu erreichen, wobei die beiden Longi sich mit dem Wachstum der neuen Fasern miteinander verschmolzen, während ihre Insertionen noch getrennt bleiben.

So wird der horizontale Longus prof: der Larve von den neuen Fasern bedeckt, die die Lage der Portio lateralis des vertikalen Longus des erwachsenen Tieres einnehmen.

2) Am Add. mand. externus (ae in Figg. 5-9).

In diesem Muskel treten die neuen Myoblasten zunächst im dorsalen Teil des Muskelbauches, und zwar gleich wie am Orbitohyoideus am frühesten unter allen Adductoren, etwa 7 Tage vor dem Durchtritt der vorderen Extremitäten. Dann wachsen die neugebildeten Fasern entlang des sich degenerierenden Fasern muskelkopf- und -schwanzwärts, während die alten Muskel- und Sehnenfasern sich auflösen.

Mit der oben erwähnten Erweichung, Zusammenschiebung und Auflösung des Proc. orbitalis während der Metamorphose, geht der Ursprung des Muskels von der Medialseite dieses Fortsatzes gegen die Basis des. selben rostralwärts hinüber. Mit der Aufrichtung des Quadratum und des Muskels erreichen die neuen Fasern gegen den sich so verschieben- 
Die Kaumuskulatur der Anuren (Bufo vulgaris japonicus) während der Metamorphose. 21

den Muskelkopf die rostrale bezw. ventrale Seite des Basis des sich auflösenden Fortsatzes und verbreiten weiter ihren Ursprung entlang der lateralen Seite desselben.

Der Externus der erwachsenen Kröte entspringt am Annulus tympanicus sowie am Proc. zygomaticus (s. o.). Wir nehmen diesen Ursprung als einen sekundären nach der Metamorphose an, wenn auch wir seine Entwicklung trotz an Hand der lückenlosen Materialreihe nicht verfolgen konnten.

Während dieser Veränderung des Muskelkopfes, verschiebt sich der Ansatz entsprechend der Verlängerung des Mandibulare caudal- und etwas lateralwärts, indem sich die alten Muskel- und Sehnenfasern degenerieren. In etwas späteren Stadien verschwindet die Sehne, wobei wir nicht meinen, daß diese Sehnendegeneration, wie Luther glaubt, mit der Entwicklung der Buccalfalte in engerem Beziehung stehe. Anderseits erreichen die neuen Fasern entlang der Fasern des sich verändernden Muskelschwanzes das Mandibulare.

So wird der Externus von den neuen Fasern bedeckt, indem der Muskel, der anfangs medial vom articularen Fortsatze des Quadratum horizontal verlief, nunmehr lateral von demselben vertikale Stellung einnimmt.

3) Am Add. mand. post. subexternus (aps in Figg. 5-10).

Im mittleren Teil dieses Muskels treten die neuen Myoblasten später als am vorigen Muskel auf, und zwar kurz nach der Entwicklung der neuen Fasern im letzteren; unter der Lupe sieht er noch gelblich aus, während der vorige schon weißliche Farbe annimmt. Gleich wie bei den übrigen Adductoren degenerieren sich die alten Muskel- und Sehnenfasern, indem die neuen Fasern muskelschwanz- und -kopfwärts wachsen, bis sie die definitive Lage annehmen.

Entsprechend der Veränderung des Proc. orbitalis, an dessen Medial. seite der Muskel entspringt, verschiebt sich der Muskelkopf gegen die rostrale bezw. ventrale Seite des Basis des sich auflösenden Fortsatzes.

Während des Durchtrittes der vorderen Extremitäten, haftet am rostralen Muskelende die Stelle, wo der Muskel in seine Sehne oder in die Sehne des Longus prof. übergeht, dem Mandibulare an. Wenn das Suprarostrale und die Sehne zugrunde gehen, setzt der Muskel sich an das Mandibulare an, und die neuen Fasern reichen während der caudalen Verschiebung des Mandibulare und der Aufrichtung des Adductor gegen den Muskelschwanz zum Mandibulare.

Am Larvenstadium liegt der gemeinsame Ansatz des Superficialis 
A. Takisawa, Y. Ōhara und K. Kanō.

und Externus an der Medialseite desjenigen des Profundus und Subexternus. Wenn die vorderen Extremitäten durchtreten, und ihre Sehnen sich an das Mandibulare anheften, ordnen sich ihre Insertions. punkte an der dorsalen Fläche des Mandibulare von medial lateralwärts in der Reihenfolge von Superf., Ext., Prof. und Subext. Entsprechend der caudalen Verschiebung des Mandibulare schieben sich die Insertionen des Prof. und Subext. zwischen diejenigen des Superf. und Ext. hinein. So wird der Subext., den man im Larvenstadium an der Ventral- und Lateralseite des Ext. sieht (Fig. 3), allmählich durch den letzteren bedeckt.

4) Am Add. mand. post. articularis ( $a p a$ in Figg. 5-10).

Erst in späteren Stadien der Metamorphose beginnt dieser Muskel sich zu erneuern. Wie bei den übrigen Adductoren degenerieren sich die alten Fasern, indem die neuen Fasern gegen den Muskelschwanz sowie -kopf heranwachsen. Währenddessen stellt er sich vor dem Quadratum vertikal, nachdem er vorhin parallel an der Medialseite des Proc. articularis quadrati verlaufen ist.

Am dorsalen Muskelende verschiebt sich der Ursprung entsprechend der Veränderung des Proc. orbitalis unter diesen Fortsatz. Die neuen Fasern reichen so entlang der schon bindegewebsstrangartig gewordenen alten Fasern des Muskelkopfes bis zum Ursprungspunkt.

Am ventralen Muskelende reichen die neuen Fasern entlang der sich auflösenden Fasern muskelschwanzwärts zum Mandibulare, das sich caudalwärts verlängert. Währenddessen findet die Umordnung der Insertionen der übrigen Adductoren statt, wobei seine Insertion ihre definitive Lage annährend zwischen dem Superf. und.Prof. einnimmt.

5) Am Pterygoideus ( $m p t$ in Figg. 5-10).

An diesem Muskel erneuern sich die Fasern auch während der Metamorphose, die Sehne bildet sich aber in etwas anderer Weise zurück, worauf wir in einer späteren Abhandlung näher zurückkommen wollen.

Die oben angegebenen Adductoren erneuern sich nicht $z u$ gleicher Zeit, sondern nacheinander. Zuerst erscheinen die neuen Myoblasten im Ext., dann im Surerf., Prof. und Pterygoideus, zuletzt im Subext. und Articularis. Diese Reihenfolge scheint uns mit der Erweichung der Skeletteile, an welchen sie entspringen, in engerem Zusammenhang $\mathrm{zu}$ stehen; z. B. entsprechend der Erweichung des Proc. orbitalis, an 
Die Kaumuskulatur der Anuren (Bufo vulgaris japonicus) während der Metamorphose. 23 welchem der Ext. und Orbitohyoideus entspringen, erscheinen die neuen Myoblasten auch in ihnen, was aber noch weiterer Untersuchung nötig ist. Wie es auch immer sei, degenerieren sich die alten Fasern in dieser Reihenfolge, und die Myofibrillen der neuen Fasern erhalten die Querstreifung auch in gleicher Reihenfolge.

Im Larvenstadium inserieren der Superf. und Ext. medial vom Prof. und Subext., der Articularis und Pterygoideus dagegen lateral von ihnen, und zwar diese drei Muskelpaare jedes für sich voneinander getrennt. Wenn die vorderen Extremitäten durchtreten, finden die Muskeln ihre Insertionen an der dorsalen Fläche des Mandibulare von medial lateralwärts in einer Reihenfolge von Superf., Ext., Prof., Subext., Pterytoideus und Articularis, wobei sie noch getrennt bleiben.

Mit der Caudalwärtsverlängerung des Mandibulare, wachsen die neuen Fasern der genannten Muskeln bis zum Mandibulare. Gegen Ende der Metamorphose nähern sich ihre Insertionen allmählich miteinander und kommen direkt rostral vom Kiefergelenk. Diese Veränderung der Insertionen geschieht wahrscheinlich durch die Verlängerung des Mandibulare während der Metamorphose; der mediale Teil des Mandibulare verschiebt sich dabei mehr caudalwärts als der laterale Teil desselben.

\section{Die Entwickl:ung des Add. mand. post. lateralis.}

(apla in Figg. 5-9).

Luther gibt bezüglich dieses Muskels der larvalen Kröte einige Beschreibungen und eine Abbildung an. Wir konnten ihn auch durch Mikrodissektion bei der Larve kurz vor dem Extremitätendurchtritt in der von Luther gesprochenen Lage finden. Mikroskopisch konnten wir auch bestätigen, daß er nur aus jungen Muskelfasern besteht. Sie stehen aber mit den larvalen Muskelfasern in keinem Zusammenhang, sondern sind von gleicher Natur wie die oben besprochenen neuen Muskelfasern, die von den Fibroblasten $z$ wischen den alten Fasern abstammen.

In diesem Stadium zieht der Muskel fast horizontal, von der lateralen Seite des rostralen Endes des Proc. articularis des Quadratum gegen die dorsalen Seite des articularen Teils des Mandibulare, wo der Articularis inseriert. Je weiter die Metamorphose vor sich geht, desto mehr wächst er heran und wird entsprechend der caudalen Verschiebung 
A. Takisawa, Y. Ōhara und K. Kanō.

des Mandibulare sowie der Aufrichtung des Quadratum vertikal, sodaß er die definitive Lage des Lateralis einnimmt.

Am Tag des Extremitätendurchtrittes sieht dieser kleine Muskel weißlich aus, was bedeutet, daß er aus jungen Fasern besteht. Kurz vor diesem Stadium besteht er mikroskopisch aus den älteren Myoblasten, welche von den umgebenden Gewebszellen leicht unterscheiden lassen. Dadurch und auch durch ihre Lage gegen das Kiefergelenk und den $\mathrm{N}$. mandibularis können wir ihre Herkunft von den am articularen Teil des Mandibulare dorsolateral befindlichen Mesenchymzellen mit Sicherheit verfolgen.

Kurz vor dem Durchtritt der vorderen Extremitäten entwickeln sich diese Mesenchymzellen schnell zu Myoblasten und dann $z \mathfrak{u}$ jungen Fasern, so daß dieselben als solche unter Lupe erkannt werden können.

Bei der Larve etwa 10 Tage vor dem Extremitätendurchtritt besitzen diese Zellen noch keine Myofibrillen; in ihrem Zytoplasma ist die basophile Substanz spärlich nachweisbar, das Glykogen dagegen ziemlich reichlich; die Mitosen sind nur in diesem Stadium beobachtet, sonst ausschließlich nur die Amitosen. Schon in diesem Stadium scheinen die Nervenfasern des Astes des N. mandibularis mit diesen Zellen in Verbindung zu stehen, was noch Gegenstand einer eingehenden Untersuchung darbietet.

Im folgenden Stadium werden die Zellen größer; ihr Zytoplasma nimmt an Menge zu und wird stärker metachromatisch; die Mitochondrien werden nur noch undeutlich beobachtet, aber einige Körner werden schon aufgefunden, das Glykogen wird dagegen ziemlich deutlich bewiesen; aber die Myofibrillen erscheinen noch nicht. Die Zellen besitzen in folgenden Stadien Myofibrillen ohne Querstreifung in ihrem metachromatischen Zytoplasma, so daß sie ja schon als echte Myoblasten aufzufassen sind; sie tragen einen oder zwei elliptische Kerne, oder weisen amitotische Kernteilung auf ; die stäbchenförmigen Mitochondrien ordnen sich um die Kerne; das Glykogen wird geringer. Mit der Streckung dieser Myoblasten vermehren sich in ihnen die Myofibrillen ohne Querstreifung an Zahl, und die Kerne auch durch amitotische Teilung.

Es ist aber uns noch nicht klar, wie diese Myoblasten den zukünftigen Lateralis bilden, indem sie als Ursprung die laterale Seite des rostralen Endes des Proc. articularis des Quadratum und als Ansatz die dorsale Seite des artikularen Teils des Mandibulare erreichen. 
Der M. intermandibularis anterior (M. submentalis Gaupps) der erwachsenen Kröte, welcher sich an den rostralen Rand des M. intermandibularis posterior (M. submaxillaris Gaupps) anschließend, im rostralsten Winkel des Unterkiefers liegt; ist möglicherweise Antagonist des Add. mand. post. lateralis. Daß der Intermand. ant. eine leichte Rotation des Unterkiefers, seinen ventralen Rand nach innen, bewirkt, wurde von Gaupp (1896, S. 138) bei Rana experimentell nachwiesen. Anderseits rotiert der Lateralis auch nach Gaupp (1896, S. 134) den Unterkiefer, so daß sein ventraler Rand nach außen kommt. Gaupp hebt aber nicht besonders hervor, da $\beta$ der Lateralis Antagonist des Intermand. ant. ist. Freilich wäre nötig, diese Wirkungen auch bei der Kröte nachzuprüfen. Bei den Urodelen, denen der Lateralis fehlt, würde nach Fischer (186t) und Ruge (1897) (zit. nach Luther (1914)) der an der Außenfläche des Unterkiefers weit hinabreichende Add. mand. externus den ventralen Rand des Unterkiefers auswärts rotieren, der Intermand. ant. dagegen einwärts. Lubosch (1938, S. 1038) schließt sich dieser Meinung an, indem er sagt: „Möglicherweise wirkt er (Intermand. ant.) bei den Urodelen ähnlich, als Antagonist des ‥M. add. mand. ext."

Nach Goette (1875) und Schulze (1892) ist der Intermand. ant. bei den Anurenlarven vorhanden. Nach Lubosch (1938) ist er auf früheren Stadien der Larve paarig, erreicht bald seine endgültige Größe und wächst während der Metamorphose nur wenig. Aber nach unser Befund an der Kröte entwickelt sich dieser Muskel während der Metamorphose gleich wie der Lateralis, worauf wir in einer späteren Abhandlung näher zurückkommen wollen.

So meinen wir, daß der Lateralis sich in engerem Zusammenhang mit der Entwicklung des Intermand. ant., seines Antagonisten entwickelt. Warum entwickelt sich aber dieser Lateralis allein unter erwachsenen Kaumuskeln während der Metamorphose ganz neu, ist unklar.

Wie schon bekannt, entstehen die Extremitäten- und Seitenbauchmuskulaturen der Anuren durch lokale Differenzierung aus dem. Mesenchym. Kaestner (1893) glaubt, da $B$ die Myoblasten sich dabei von den ventralen Myotomkanten ablösen und als Stammzellen der betreffenden Muskeln dem Mesenchym der Anlagen beigesellen, was aber bisher nicht nachgewiesen ist. Nach Schaffer (1933, S. 204) sind selbst beim Neugeboren der Säugetiere nicht alle Myoblasten aufgebraucht, und man findet an der Oberfläche mancher Muskeln noch Reservematerial, aus 
dem eine Art appositionellen Wachstums der Muskeln durch Neubildung von Muskelfasern stattfindet. Anderseits bestätigt Katznelson (193435) bei Urodelen den mesenchymatösen Ursprung der Muskeln der Extremitäten, der Kiemen, der Bauchwand und des Kopfes. Nach Lubosch (1937) sollen solche mesenchymatösen Zellen wohl Reservemyoblasten sein.

Obwohl die ursprüngliche Herkunft der Mesenchymzellen sowie der Fibroblasten des Endomysiums bei unserem Fall auch noch Gegenstand eingehenderer Untersuchungen ist, könnten wir diese zwei Arten Myogenesen vielleicht wohl mit den Osteogenesen bei Beleg- und Ersatzknochen verglichen werden.

\section{Zusammenfassung.}

Zuerst haben wir die Kaumuskeln der erwachsenen und larvalen Kröte zur Ergänzung des Lutherschen Befundes beschrieben, und das Vorkommen des von Luther vermissten $M$. add. mand. post. subexternus bestätigt.

Wir haben die Entwicklung der Kaumuskeln während der Metamorphose bei der Kröte studiert; wir wendeten dabei Mikrodissektion und Schnittuntersuchung mit verschiedenen Fixierungen (Levi, Regaud, Bou in, Zen ker-Formol, Formol-Alkohol) und verschiedenen Färbungen (Hämatoxylin-Eosin, Thionin, Heidenhain's Eisenhämatoxylin, Bauersche Glykogenfärbung, Überjodsäure-Schiffsche Reaktion, Feulgensche Nuklealreaktion, Azan) an und kamen zum Schlusse, daß die Kaumuskeln der Kröte während der Metamorphose sich in folgender Weise umformen:

a) Die larvalen Kaumuskeln werden durch die neuen Muskelfasern ersetzt, indem die horizontal gelagerten Muskeln nunmehr vertikale Lage annehmen-Adductor mandibulae externus, Add. mand. post. subext., Add. mand. post. longus superf.(=Portio medialis des erwachsenen Add. mand. post. long.), And. mand. post. long. prof. (= Portio lateralis desselben), Add. mand. post. articularis und Pterygoideus, von denen der zuletzt genannte Muskel sich etwas anders als die übrigen verhält, worauf wir in einer späteren Abhandlung näher zurückkommen wollen. 1) Am Eintritt der Metamorphose erscheinen die neuen Myoblasten, die von den Fibroblasten des Endomysiums der larvalen Kaumuskeln abstammen und zwar zunächst im mittleren Teil derselben.

2) Im Laufe der Metamorphose wachsen die neuen Muskelfasern, die sich von den neuen Myoblasten entwickeln, und sich entlang des sich 
Die Kaumuskulatur der Anuren (Bufo vulgaris japonicus) während der Metamorphose. 27

degenerierenden alten Fasern muskelschwanz- und -kopfwärts oder dann entlang des sich auflösenden Knorpels verlängern, um den neuen Ursprung und Ansatz zu gewinnen.

3) Die alten Fasern gehen dagegen alle zugrunde.

4) Die zytologische Natur der neuen Myoblasten und

5) die Degenerationsvorgänge der alten Muskelfasern sind gleich, wie bei Entwicklung des Depressor mandibulae während der Metamorphose (Takisawa und Sunaga, 1951).

6) Die Sehnen des Larvenstadiums auch gehen zugrunde.

7) Wir haben an einzelnen Muskeln die Verschiebung der neuen Muskelfasern gegen ihre definitive Lage verfolgt.

8) Die Umformung der einzelnen Muskeln geschieht nicht zu gleicher Zeit, sondern nacheinander.

b) Anderseits entwickeln sich um den artikularen Teil des Mandibulare Mesenchymzellen, die mit den larvalen Kaumuskeln in keinem Zusammenhang stehen, und Myoblasten liefern, aus welchen ein Kaumuskel des erwachsenen Tieres entsteht, nämlich der Add. mand. post. lateralis. Dieser Muskel entwickelt sich zu gleicher Zeit mit dem Intermand. ant., seinem Antagonisten erst während der Metamorphose.

Zum Schluß sprechen wir Herrn Prof. Dr. S. Nishi, Rektor unserer Universität, für seine Ermutigung bei unserer Arbeit, Herrn Prof. Dr. T. Itô, Vorstand unseres Anatomischen Institutes, für seine wertvollen Ratschläge hinsichtlich der Zytologie, und Herrn Prof. Dr. T. Katsumata, Direktor des Anatomischen Institutes der Medizinischen Hochschule zu Yokohama, für seine stete liebenswürdige Unterstützung unseren herzlichsten Dank aus.

\section{Literaturverzeichnis.}

Edgew orth, F. H., 1911, On the Morphology of the cranial muscles in some Vertebrates. Quart. Journ. of microsc. Sc., vol. 56. (N. S. No. 222).

Ders., 1935, The cranial muscles of Vertebrates. Cambridge.

Gaupp, E., 1893, Beitr. z. Morphologie d. Schädels. I : Primordialcranium u. Kieferbogen v. Rana fusca. Morphol. Arbeiten (Schwalbe) II.

Ders., 1896, Eckers u. Wiedersheims Anatomie d. Frosches. 1. Abt. 3. Aufl., Braunschweig. Go ette, A., 1875, Die Entwicklungsgeschichte d. Unke. Leipzig.

Kaestner, S., 1893, Die Entwicklung d. Extremitäten- u. Bauchmuskulatur b. d. anuren Amphibien. Arch. f. Anat. u. Phys., Anat. Abt.

Katsumata, $T$, 1938, Identifizierungen d. Kopfmuskeln d. Anurenlarven $m$. denen d. erwachsenen Tieres. I. Mitt. Über d. Entwicklung d. von Facialis innervierten Muskeln b. Kröten. Kaibo Z., 12. (Japanisch). 
Katznelson, Z.S., 1934, Über d. mesenchymatöse Entwicklung d. quergestreiften Muskeln b. Amphibien. C.r. Ac. Sci. Leningrad, 2. (zit. n. Katznelson, 1934b u. n. Anat. Ber. 33.)

Ders., 1934b, Histogenesis of muscular tissue in Amphibia. 1. Development of striated muscles from mesenchyma in Urodeles. Anat. Rec. vol. 61.

Ders, 1935, Uber d. Entwicklungsquelle d. latero-ventralen Teiles d. Rumpfmuskeln sowie d. caudalen Muskulatur b. Amphibien. C.r. Ac. Sci. Leningrad, 2, 4. (zit. n. Anat. Ber. 34, 36.)

Kawai, T., 1942, Die normale Entwicklung d. Kopfmuskulatur v. Anuren im Laufe d. Metamorphose. I. Mitt. Über d. Entwicklung d. von Facialis innervierten Muskeln b. Fröschen. Kaibo Z., 19. (Japanisch).

Lubosch, W., 1913, Die Kaumuskulatur d. Amphibien, verglichen m. d. der Sauropsiden u. Säugetiere. Anat. Anz., Ergh. zu Bd. 44. (Verh. Anat. Ges. Greifswald).

Ders., 1915, Vergl. Anatomie d. Kaumuskeln d. Wirbeltiere. I. Die Kaumuskeln d. Amphibien. Jena. Zschr. Naturw. 53, N. F. 46.

Ders., 1937, Muskel u. Sehne. Morphol. Jb., 80.

Ders., 1938, Handb. d. vergl. Anatomie. V.

Luther, A, 1914, Über d. vom N. trigeminus versorgte Muskulatur d. Amphikien. Acta Soc. Sci. Fenniciae, Vol. XLIV. No. 7.

Maurer, F., 1915, Grundzüge d. vergl. Gewebelehre. Leipzig.

Müller, H., 1921, Die dorsale Stammuskulatur d. Frosches während d. Metamorphose. Zschr. wiss. Zool. 118.

Sch a f fer, J., 1933, Lehrb. d. Histol. usw. 3. Aufl. Leipzig.

Schulze, F. E., 1892, Über d. inneren Kiemen d. Batrachierlarven. II. Mitt. Abh. Akad. d. Wiss. Berlin.

Takisawa, A. u. Sunaga, Y., 1951, Über d. Entwicklung d. M. depressor mandibulae b. Anuren im Laufe d. Metamorphose. Folia anat. jap. 23.

Takis awa, A. u. Kanō, K., 1951, Über d. Glykogen im Gallertgewebe b. Anurenlarven. Kaibo Z. 26. (Japanisch). 